Technical Note: Explaining Variance of Pleistocene Climate Sensitivity - Not Peer Reviewed

\title{
Explaining Variance of Pleistocene Climate Sensitivity: Path, Epochal and $\mathrm{CO}_{2}$ dependence
}

\author{
Roger M. Cooke ${ }^{1}$, Willy P. Aspinall ${ }^{2}$ \\ ${ }^{1}$ Resources for the Future, Washington DC, 200036 USA (cooke@rff.org) \\ ${ }^{2}$ School of Earth Sciences, University of Bristol, Bristol, UK (willy@aspinall.associates) \\ Jan 23, 2022 \\ Correspondence to: Roger M. Cooke (cooke@rff.org) \\ http://rogermcooke.net/
}




\title{
Explaining Variance of Pleistocene Climate Sensitivity: Path, Epochal and $\mathrm{CO}_{2}$ dependence
}

\author{
Roger M. Cooke ${ }^{1}$, Willy P. Aspinall ${ }^{2}$ \\ ${ }^{1}$ Resources for the Future, Washington DC, 200036 USA \\ ${ }^{2}$ School of Earth Sciences, University of Bristol, Bristol, UK \\ Jan 23, 2022 \\ Correspondence to: Roger M. Cooke (cooke@rff.org)
}

\begin{abstract}
Improved high-resolution paleo records of atmospheric carbon dioxide $\left(\mathrm{CO}_{2}\right)$ concentrations and reconstructions of Earth's surface temperature are available. We analyse one authoritative Pleistocene dataset to explore how the climate sensitivity parameter $S$ varies under different system states, using linear regression of mean annual surface temperature changes against $\mathrm{CO}_{2}$ forcing changes. On the whole data set, $\mathrm{S}=2.04 \mathrm{~K} / \mathrm{Wm}^{-2}$ and $\mathrm{CO}_{2}$ forcing explains $64 \%$ of the variance in temperature. Partitioning the data by path (glaciation / deglaciation); during deglaciation episodes, $S=2.34 \mathrm{~K} / \mathrm{Wm}^{-2}$, explaining $75 \%$ of the temperature variance; during glaciations, $S=1.59 \mathrm{~K} / \mathrm{Wm}^{-2}$ explaining $48 \%$ of the temperature variance. Further partitioning into epochs before and after the Marine Isotope Stage $11424 \mathrm{kaBP}$ (thousand years before present); for deglaciation paths after $424 \mathrm{kaBP}, S=2.63 \mathrm{~K} / \mathrm{Wm}^{-2}$ explaining $83 \%$ of the variance. Partitioning into levels of $\mathrm{CO}_{2}$ concentration has much less explanatory power; $20 \%$ for concentrations above $210 \mathrm{ppmv}$ and $2 \%$ for concentrations below $210 \mathrm{ppmv}$. These partitions conflate glaciation and deglaciation episodes and pre/post $424 \mathrm{kaBP}$ epochs. Possible process-related explanations for the path-related differences are conjectured. The common assumption that changes in temperature are proportional to changes in $\mathrm{CO}_{2}$ forcing constrains the regression intercept to pass through the origin and inflates the regression error to the extent that the regression model becomes a worse predictor of temperature changes than simply predicting the population mean for all values of $\mathrm{CO}_{2}$ forcing. The consequences of this assumption are substantive in the context of estimating future temperature trajectories and should be carefully weighed.

Key words: climate sensitivity parameter, glaciation, deglaciation, explained variance, regression intercept
\end{abstract}

\section{Introduction}

The climate sensitivity parameter $S$ is somewhat loosely defined in the literature (Myhre et al 2013) as the change with respect to present in mean annual global surface temperature $(\Delta T)$ per unit change in forcing with respect to present $(\Delta F)$. $S$ is sometimes estimated from data by linear regression:

$\Delta T=S \times \Delta F+B+e$

with intercept $B$ and error $e . \Delta F$ is $\left[W^{-2}\right]$ so that $S$ is $\left[K /\left(W m^{-2}\right)\right]$. The variance of the error $e$ is estimated as $(S S-S S R) /(N$ $-d f-1$ ) where $N$ is the number of observations, $d f$ is the number of parameters in the model (here always $=1$ ), $S S$ is the sum square deviations from the population mean and $S S R$ is the sum square deviations of the regression estimates from the population mean. $S S R / S S$ is the fraction of variance of $\Delta T$ explained by the regression model, termed $R^{2}$. The lower is $R^{2}$ the more of the variation in $\triangle T$ must be attributed to factors other than $\triangle F C O_{2}$. If $S S R=S S$ then the regression model explains all the variance in the data. If $S S R>S S$ then the regression predicts $\Delta T$ with a variance that is greater than the population variance; in other words, the model predictions are worse than simply predicting the population mean of $\Delta T$ for all values of $\Delta F$.

It is widely believed that “... the change in surface temperature is directly proportional to the radiative forcing. Hence, this becomes the simplest way of quantifying the effect in a perturbation in greenhouse gas inventory" (Byrne and Goldblatt 2014). Others argue that the regression line "needs to pass through the origin to avoid any biases" (Köhler et al 2017). Note that $\Delta T$ can be proportional to $\Delta F$ only if the intercept $B$ is zero. Assuming $B=0$ forces the trend line to pass through the 
origin $(\Delta F=0, \Delta T=0)$. This can inflate and skew the errors and can cause the regression model to be a worse predictor of $\Delta T$ than simply predicting the mean of $\Delta T$ for each value of $\Delta F$. This actually happens with the dataset under analysis (see Figure 1 and associated discussion). Different statistical packages have different interpretations of $R^{2}$ when $B=0$ is stipulated, but none has the interpretation as fraction of explained variance. Indeed, if $S S<S S R$, then variance estimate of $e$ as $(S S-S S R) /(N-d f-1)$ would be negative. In what follows, we refrain from stating $R^{2}$ values if $B$ is set to zero. Of course, the placement of the origin effects the value of $S$ if $B=0$ is stipulated. For example, Snyder (2019) considers $\Delta T$ relative to the average temperature over the last five thousand years, whereas Martinez-Boti et al (2015) define temperature change relative to pre-industrial temperature. If on the other hand the intercept is estimated this choice is immaterial. In any event, the consequences of stipulating $B=0$ are substantial and should be carefully weighed.

Here, we utilize the dataset (Martinez-Boti et al 2015) for the Pleistocene (1096 records from 0.14 to $798.51 \mathrm{kaBP}$ (thousands of years before present). A second Pleistocene dataset (Snyder, 2019) yields very similar results and is discussed briefly. In recent years, several researchers have studied paleoclimate data for evidence of "state dependence" in the climate sensitivity parameter (e.g. Meraner et al 2013). It has been observed that the linear approximation breaks down in the long tail of high climate sensitivity commonly seen in observational studies (Bloch-Johnson et al 2015). Transient behavior of climate sensitivity has been explored using an energy balance model combined with observational and modeling CMIP5 constraints (Goodwin 2018). Background state dependence and tipping points in Earth system sensitivity with millennial timescales (von der Heydt et al 2016) motivate the introduction of the more general "climate sensitivity parameter". Others find that climate sensitivity strongly depends on the climate background state, with significantly larger values attained during warm phases (Meraner et al 2013. Friedrich et al 2016).

However, some authors voice concerns over the simple concepts underlying climate sensitivity and radiative forcing (Knutti et al 2010). Non-linear dependence of land ice albedo forcing is found (Köhler et al 2010), while non-linearity of $\mathrm{CO}_{2}$ forcing is said to depend on the $\mathrm{CO}_{2}$ data set. Global mean temperature and $\mathrm{CO}_{2}$ diverge during intervals of pronounced land ice growth (Köhler et al 2018). The need to distinguish actuo- and paleoclimate sensitivity over different time scales is emphasized (Rohling et al 2018). Averaged glacial and interglacial climate sensitivities are estimated (Shao et al 2019) using Earth system model simulations of the Last Glacial Cycle.

The present study applies standard regression analysis to study variations in the climate sensitivity parameter, using Pleistocene data from Martínez-Botí et al (2015). Rather than estimating non-linear and/or multivariate regression functions, we partition the data into periods of increasing versus periods of decreasing paths of $\mathrm{CO}_{2}$ concentration corresponding to deglaciation versus glaciation respectively. Table 1 also looks at partitions into epochs before and after $424 \mathrm{kaBP}$ and periods of low, intermediate and high $\mathrm{CO}_{2}$ concentration. These partitions retain ample statistical power. Path dependence is the most important followed by epochal dependence. Dependence on background $\mathrm{CO}_{2}$ concentration has low explanatory power but does interact with the other two.

To compute the fraction of explained variance it is necessary to estimate the intercept term. Comparison with regressions in which the intercept $B$ is set to zero shows that the latter have higher values of the climate sensitivity parameter with much less variation over the partition elements. While this data may be subject to errors, random errors would depress the $R^{2}$ values. The wide variation of $R^{2}$ values across the partition elements argues against a strong random or systematic error across the entire data set.

Given the wide variations in $S$, projections of regression equations to estimate $\mathrm{CO}_{2}$ concentrations out of sample cannot be considered predictions unless the conditions going forward are very similar to the conditions on which such regressions are based.

\section{Analysis of Pleistocene data}

A deterministic functional relation between changes in $\mathrm{CO}_{2}$ concentrations and changes in the induced forcing $\left(\triangle F C \mathrm{O}_{2}\right)$ is inferred from radiative transfer codes, where $278 \mathrm{ppm} v$ is taken as the pre-industrial concentration of $\mathrm{CO}_{2}$ : 
$\triangle \mathrm{FCO}_{2}\left(\mathrm{CO}_{2}\right)=5.3515 \times \ln \left(\mathrm{CO}_{2} / 278\right)\left[\mathrm{Wm}^{-2}\right]$.

Note that the forcing change depends only on the ratio of two $\mathrm{CO}_{2}$ concentrations and not on their actual values. This assumption is pervasive in the literature. Doubling $\mathrm{CO}_{2}$ with respect to pre-industrial:

$\triangle \mathrm{FCO}_{2}(556)=3.71\left[\mathrm{Wm}^{-2}\right]$.

Regressing mean annual surface temperature ( $\triangle M A T$ in Martinez-Boti et al 2015) on $\triangle F C \mathrm{O}_{2}$ gives the following equation which explains $64 \%$ of the variance in $\Delta T$ :

$\Delta T\left(\Delta F C \mathrm{O}_{2}\right)=2.037 \times \Delta \mathrm{FCO}_{2}-0.709+e$

The climate sensitivity parameter $S=2.037\left[\mathrm{~K} / \mathrm{Wm}^{-2}\right]$. The values of $S$ and $B$ in eqn. (4) are chosen to minimize the squared distance between the values of $\Delta T$ and the linear trend.

To project the effect on temperature of doubling $\mathrm{CO}_{2}$ above pre-industrial, based on the whole Pleistocene data, substitute $\Delta F C O_{2}=3.71 \mathrm{in}(4)$ and find $\Delta T(556)=+6.85^{\circ} \mathrm{K}$. Neglecting the intercept term would result in $2.037\left[\mathrm{~K} / \mathrm{Wm}^{-2}\right] \times 3.71[\mathrm{~W}]$ $=+7.56^{\circ} \mathrm{K}$. This, of course, is incorrect: if we wish to constrain the intercept to be zero, we must find the line passing

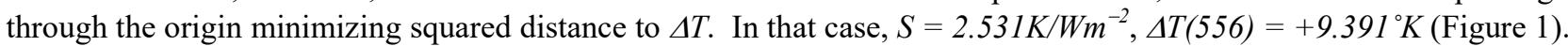

Eqn. (2) is just a positive affine transformation of $\ln \left(\mathrm{CO}_{2}\right)$. If we regress $\Delta T$ on $\ln \left(\mathrm{CO}_{2}\right)$, we would also explain $64 \%$ of the variance and also find $\Delta T(556)=+6.85^{\circ} \mathrm{K}$. The only difference would be that the units of the linear coefficient would be $[K / \ln (p p m v)]$, instead of $\left[K / W^{-2}\right]$. The latter dimensioning suggests physical agency; indeed, a $G H G$ induced radiative imbalance at the top of the atmosphere causes warming according to the Stefan Boltzmann law. At the same time, a rise in temperature can raise atmospheric $\mathrm{CO}_{2}$ concentrations through temperature mediated feedbacks as, for example, when higher ocean temperatures reduce $\mathrm{CO}_{2}$ uptake in the oceans. We retain the familiar dimension of $\mathrm{K} / \mathrm{Wm}^{-2}$ for the climate sensitivity parameter while recognizing that it reflects a choice of units rather than physical agency.

For the Pleistocene data of Martinez-Boti et al (2015), the mean $\Delta T=-2.816^{\circ} \mathrm{K}$. Letting $i$ index the 1096 data points, summing the squared deviations from the mean of the black regression line $(B=0)$ in Figure 1 gives $\operatorname{SSR}=\Sigma_{i}(2.531 \times$ $\left.\Delta \mathrm{FCO}_{2}(i)+2.816\right)^{2}=2969>2942=\Sigma_{i}(\Delta T(i)+2.816)^{2}=S S$. Using the black line as a predictor of $\Delta T$ is a bit worse than predicting the population mean of $\Delta T$ for each value of $\triangle F C O_{2}(i)$. For the red regression line ( $B$ estimated), $S S R=\Sigma_{i}$ $\left(2.037 \times \Delta \mathrm{FCO}_{2}(\mathrm{i})-0.709+2.816\right)^{2}=1896$, which explains $1896 / 2942=0.644(64 \%)$ of the variance of $\Delta T$.

Neglecting to clarify whether the intercept is inferred or stipulated invites confusion. With this dataset, setting $B=0$ inflates the $S$ values and suppresses the differences over different partition elements. Table 1 compares results with and without the intercept assumption. 


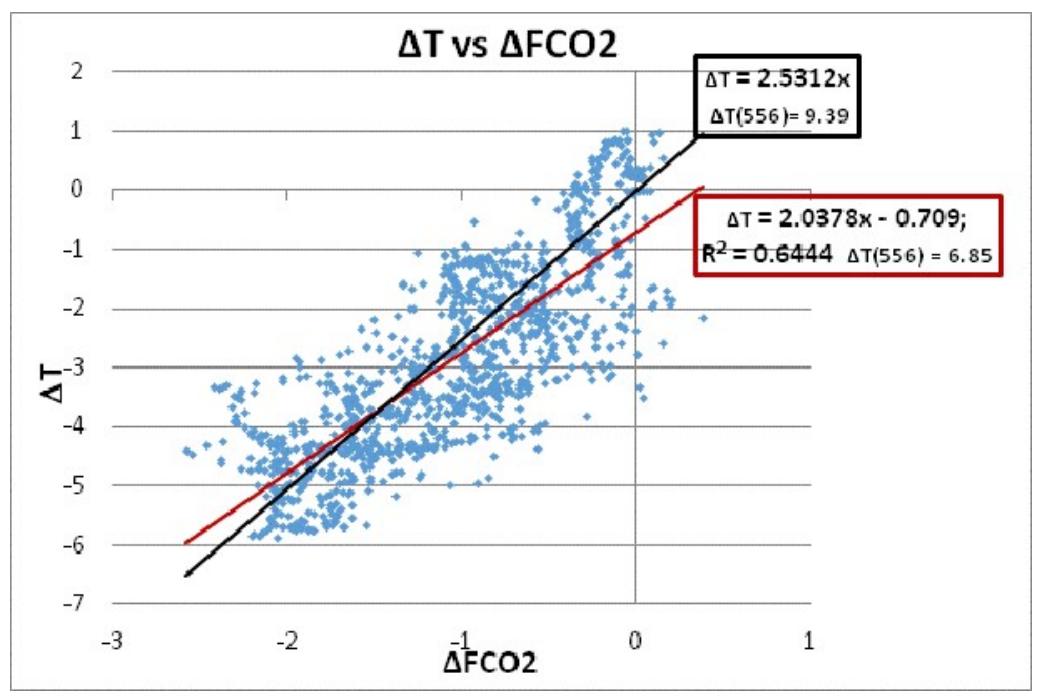

Figure 1: Regression of $\triangle T$ on $\triangle F C \mathrm{O}_{2}$ for all Pleistocene data; with (red) and without (black) intercept. Out of sample values for doubling pre-industrial $\mathrm{CO}_{2}, \Delta T(556)$ are also shown.

\section{Partitioning the data}

Figure 2 shows $\mathrm{CO}_{2}$ as a function of age in $k a B P$, with local minima and maxima identified with blue resp red arrows. This enables us to distinguish episodes of increasing (deglaciation) and decreasing (glaciation) $\mathrm{CO}_{2}$ concentrations. Note that increasing $\mathrm{CO}_{2}$ episodes generally transpire more quickly than decreasing $\mathrm{CO}_{2}$ episodes. Dividing the data into two subsets consisting of increasing or decreasing episodes allows us to determine whether the climate sensitivity parameter is $\mathrm{CO}_{2}$ path dependent. Figure 2 also suggests that the Earth's climate system changed around the inception of Marine Isotope Stage 11 in $424 \mathrm{kaBP}$, midway between a glacial maximum and a glacial minimum. Table 1 compares sensitivity in the periods before and after $424 \mathrm{kaBP}$.

The results show evidence of path dependence. Note that if the intercept is estimated the differences in the climate sensitivity parameter between glaciation and deglaciation $(1.5885,2.3352)$ are larger than if the intercept is stipulated to be zero $(2.5051,2.552)$. The same is true for the projected temperature at 522ppmv $\mathrm{CO}_{2}$ : with the intercept estimated these are $(4.72,8.32)$ for glaciation and deglaciation but with intercept stipulated these are $(9.16,9.47)$. The right panel of Figure 3 showing glaciation also illustrates how stipulating the intercept can lead to inferior predictions. Summing over the 583 data points in the right panel, the square differences between $\Delta T$ and the prediction with intercept $=0$ (red line) is 674.1 , whereas this sum is (blue line) 478.7 when the intercept is estimated. Note also the difference in explanatory power in the two panels. During deglaciation the blue regression line explains three fourths of the variance in $\Delta T$ whereas during glaciation less than half of the variance is explained. The interpretation is that during glaciation factors other than $\mathrm{CO}_{2}$ account for the variation in $\Delta T$. 


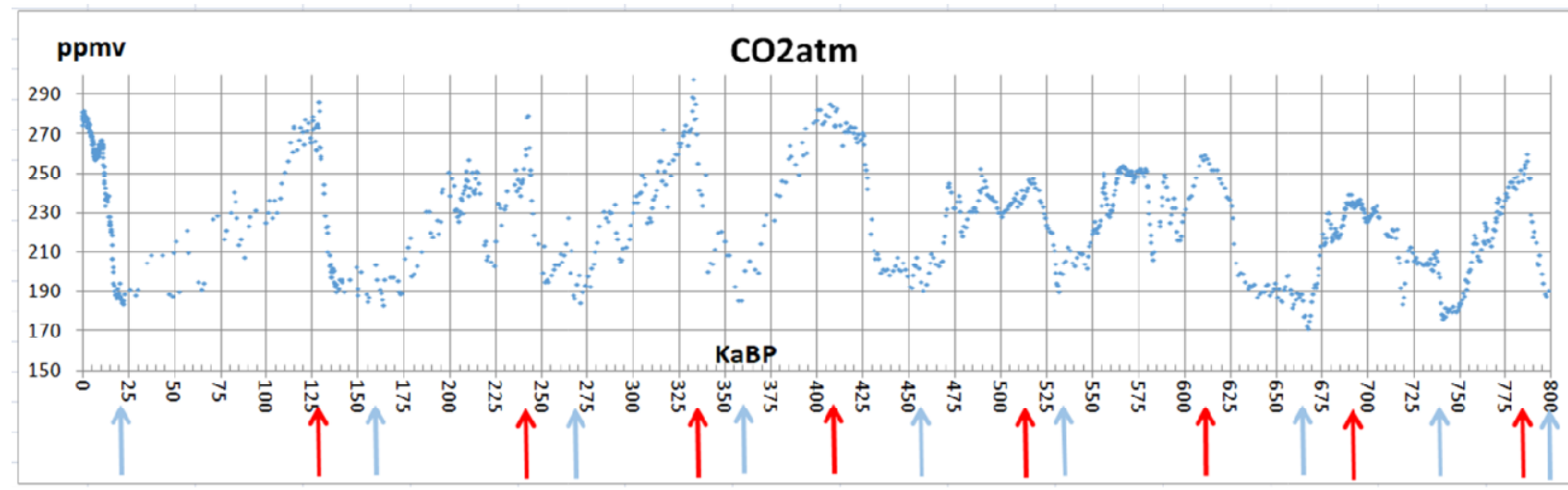

Figure 2: CO2 concentrations as function of kaBP. Blue arrows indicate local minima, red arrows indicate local maxima.
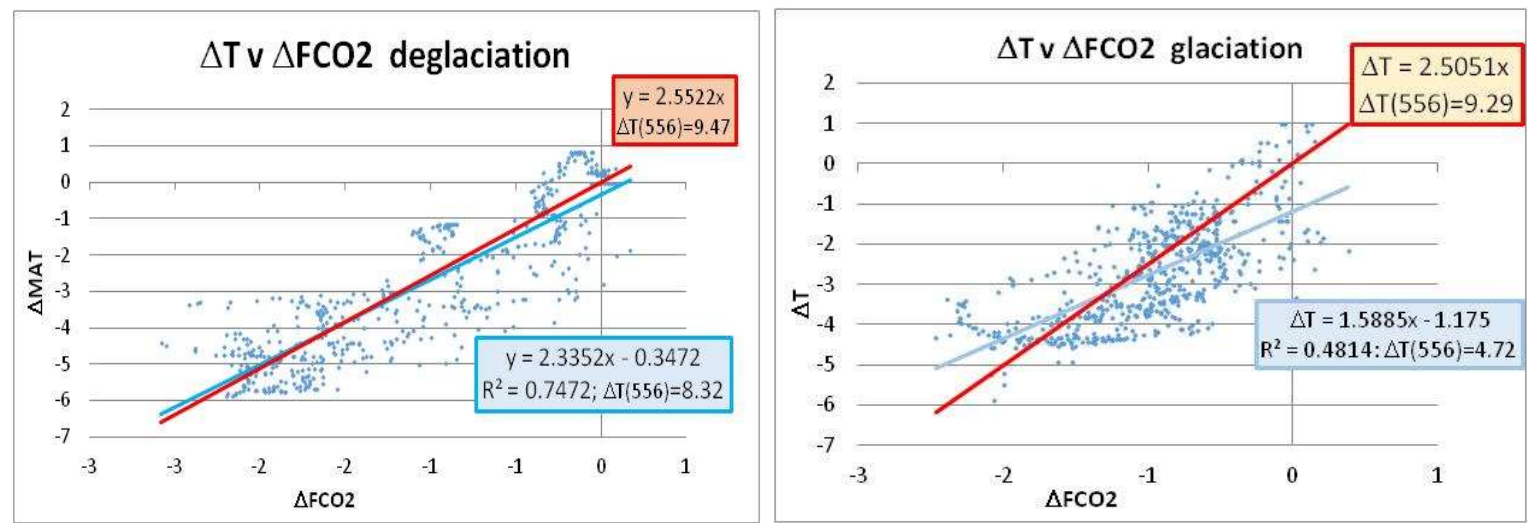

Figure 3: Deglaciation, (left, increasing) and glaciation (right, decreasing) CO2 paths. Regression for B=0 stipulated in Red, with B estimated in Blue.

A more recent study (Snyder 2019) presents a Pleistocene data set that contains 799 records, evenly spaced in steps of 1000yr. Modeling, subjective probabilities and Monte Carlo analysis are applied in Snyder (2019) to quantify uncertainties in data and variable relationships. Changes in temperature with respect to the average over the last 5000 years are regressed on the total change in forcing $(\Delta R)$ from greenhouse gases (GHG), land ice, dust and vegetation. A good comparison with Martinez-Boti et al (2015) is obtained by considering just the medians of the various terms. With respect to Snyder (2019) data, regressing the median of $\Delta T$ (termed Global Annual Surface Temperature GAST) on the median of $\Delta F G H G$, with intercept, yields:

$\Delta T(\Delta F G H G)=1.962 \times \Delta G H G-0.788+e ; \Delta T(556)=+6.49 K$

which compares well with eqn. (4), above, especially if we consider that Snyder (2019) estimates change in temperature with respect to the average surface temperature over the last 5000 years. This is $0.45 \mathrm{~K}$ higher than the pre-industrial reference value according to the analysis of Martinez-Boti et al (2015),

Hence, we should compare $6.49+0.45=+6.94 \mathrm{~K}$ to $+6.85 \mathrm{~K}$ from eqn. (4). The medians of the separate forcing terms $\Delta R_{[G H G]}, \Delta R_{[L I]}, \Delta R_{[A E]}, \Delta R_{[V G]}$ are all highly correlated with each other and with $\Delta T$. Separating these forcing contributions means assuring that, on $1000 y r$ timescales, land ice, dust and vegetation forcings are not already bleeding into the GHG forcing. This issue is avoided by focusing just on GHG forcing. The penalty is that conclusions are wedded to the $1000 y r$ timescale. 
Summary statistics comparing results with and without $B=0$ are presented in Table 1: the partitions Pre and Post 424 kaBP are intersected with deglaciation and glaciation periods.

\begin{tabular}{|c|c|c|c|c|c|c|c|c|}
\hline & \multicolumn{5}{|c|}{ Intercept estimated } & \multicolumn{3}{|c|}{ Intercept stipulated $=0$} \\
\hline & $\mathrm{R}^{2}$ & $S$ & $\mathrm{~B}$ & SE & $\Delta T(556)$ & $\mathrm{S}$ & SE & $\Delta T(556)$ \\
\hline All (1096) & 0.644 & 2.038 & -0.709 & 0.978 & 6.851 & 2.531 & 1.047 & 9.391 \\
\hline All Pre 424 (546) & 0.419 & 1.427 & -1.535 & 0.883 & 3.760 & 2.489 & 1.082 & 9.232 \\
\hline All Post 424 (550) & 0.726 & 2.320 & -0.395 & 0.994 & 8.212 & 2.595 & 1.006 & 9.626 \\
\hline \multicolumn{9}{|c|}{ De-Glaciation (increasing $\mathrm{CO}_{2}$ ) } \\
\hline All (513) & 0.747 & 2.333 & -0.348 & 0.996 & 8.309 & 2.552 & 1.014 & 9.469 \\
\hline Pre $424(220)$ & 0.457 & 1.804 & -0.959 & 1.009 & 5.733 & 2.420 & 1.145 & 8.977 \\
\hline Post 424 (293) & 0.830 & 2.631 & -0.215 & 0.916 & 9.545 & 2.798 & 0.781 & 10.381 \\
\hline \multicolumn{9}{|c|}{ Glaciation (decreasing $\mathrm{CO}_{2}$ ) } \\
\hline All (583) & 0.481 & 1.588 & -1.175 & 0.908 & 4.718 & 2.505 & 1.076 & 9.294 \\
\hline Pre $424(326)$ & 0.352 & 1.170 & -1.833 & 0.759 & 2.507 & 2.593 & 1.061 & 9.621 \\
\hline post 424 (257) & 0.538 & 1.744 & -0.782 & 0.980 & 5.689 & 2.359 & 1.080 & 8.752 \\
\hline \multicolumn{9}{|c|}{ Low, Medium, $\mathrm{High} \mathrm{CO}_{2}$} \\
\hline$<210(305)$ & 0.022 & 0.485 & -3.576 & 0.779 & -1.778 & 2.367 & 0.902 & 8.781 \\
\hline $210 \ldots . .250(493)$ & 0.195 & 1.913 & -0.943 & 0.953 & 6.156 & 2.810 & 0.978 & 10.424 \\
\hline$>250$ (298) & 0.201 & 2.711 & -0.396 & 1.096 & 9.664 & 3.670 & 1.123 & 13.614 \\
\hline
\end{tabular}

Table 1: Summary of data subset regressions. $R^{2}$ is the fraction of variance of $\Delta T$ explained by the regression, $S$ is the climate sensitivity parameter, $B$ is the intercept, $S E$ is the standard deviation of the difference between predictions and true values, and $\Delta T(556)$ is the projected $\Delta T$ corresponding to a doubling of the pre-industrial CO2 concentration (278 ppmv). With the exception of "All" and "Pre 424" all S values are outside the $90 \%$ confidence bands of the other values within each group. The number of samples is in parentheses.

\section{Summary and discussion}

Extending the analysis of the Pleistocene climate data of Martinez-Boti et al (2015) and of Snyder (2019), one key finding is that imposing a zero intercept on the regression of mean annual surface temperature changes against $\mathrm{CO}_{2}$ forcing changes tends to mask differences between the climate sensitivity parameter in different subsets of the Pleistocene data. In terms of variance explained, the zero intercept assumption produces inferior predictions relative to regressions in which estimates of intercept are exploited. Ultimately, this constraint prevents us from appreciating explanatory power when comparing climate sensitivity parameter estimates under different physical situations.

Explaining why the climate sensitivity parameter is higher during deglaciation than during glaciation is a challenge which deserves attention. One obvious feature is the fact that deglaciation generally transpires faster than glaciation. It may be that certain negative feedbacks with intermediate time scales get played out substantially during glaciation but are less effective during rapid deglaciation. 
For example, carbon fertilization would draw down atmospheric $\mathrm{CO}_{2}$. During glaciation the retreat of plants would tend to retard the cooling and slow the glaciation process. During deglaciation the advance of plants would tend to retard the warming process. However, if the retreat of land and sea ice happened quickly, other changes, such as growth of the Hadley cells and desertification, might overwhelm the advance of plants and disable this negative feedback. Oceanic $\mathrm{CO}_{2}$ outgassing during the last deglaciation has been proposed (Shao et al 2019) as a potential disequilibrium process influencing atmospheric $\mathrm{CO}_{2}$ concentrations. Aerosols, and glacial aerosols specifically, can interact with clouds and influence radiative forcing; paleo-aerosol concentrations are likely to have varied with glaciation pathways. However, the lack of robust reconstructions of glacial aerosol forcing is a key source of uncertainty in paleo-based estimates of climate sensitivity (Friedrich, and Timmermann 2020). As a further possibility, Xie (2020) posits that non-uniform regional uptake of heat by oceans militates against equilibration with radiative forcing, with spatio-temporal variations in ocean state and currents affecting global climate sensitivity.

These are just some conceptual examples of how the interplay of process-related feedbacks with different time scales might alter climate sensitivity in different physical situations. The climate sensitivity parameter is higher after $424 K a B P$, than before. Such effects have been attributed to changes in orbital forcing, though a detailed explanation of why heightened forcing might raise climate sensitivity has not, to our knowledge, been found.

If the Earth's surface temperature response to $\mathrm{CO}_{2}$ forcing on millennial time scales does indeed depend on the physical circumstances at the time of the forcing, predictions for the Earth's future response to heightened $\mathrm{CO}_{2}$ forcing require knowledge of these complex non-linear interactions, an understanding of how they will evolve and on what timescales. Scrutiny and clarification of assumptions regarding the intercept issue and further analytical investigation with stochastic uncertainty techniques should provide additional insights into the properties of the climate sensitivity parameter and inform discussion of contingent implications.

Acknowledgments The authors are grateful to Gavin Foster and Tom Gernon for subject matter advice, and to Gordon Woo for commenting on a draft manuscript.

\section{References}

Bloch-Johnson, J., Pierrehumbert, R. T. \& Abbot, D. S. Feedback temperature dependence determines the risk of high warming. Geophys. Res. Lett. 42, 4973-4980, doi:10.1002/2015GL06424 (2015).

Byrne, B. \& Goldblatt, C. Radiative forcing at high concentrations of well-mixed greenhouse gases. Geophys. Res. Lett. 41, 152-160, doi:10.1002/2013GL058456 (2014).

Friedrich, T. \& Timmermann, A. Using Late Pleistocene sea surface temperature reconstructions to constrain future greenhouse warming. Earth Planet. Sci. Lett. 530, 115911 doi: 10.1016/j.epsl.2019.115911 (2020).

Friedrich, T. et al. Nonlinear climate sensitivity and its implications for future greenhouse warming. Sci. Adv. 2, e1501923 (2016).

Goodwin, P. On the time evolution of climate sensitivity and future warming. Earth's Future 6, 1336-1348, doi:10.1029/2018EF000889 (2018),

Knutti, R., Rugenstein, M. A. A. \& Hegerl, G. C. Beyond equilibrium climate sensitivity. Nature Geoscience 10, 727-736; doi: 10.1038/NGEO3017 (2017).

Köhler, P. et al. A state-dependent quantification of climate sensitivity based on paleodata of the last 2.1 million years. Paleoceanography 32, 1102-1114; https://doi.org/10.1002/2017PA003190 (2017).

Köhler, P. et al. The effect of obliquity-driven changes on paleoclimate sensitivity during the late Pleistocene. Geophys. Res. Lett. 45, 6661-6671; doi:10.1029/2018GL077717 (2018).

Köhler, P. et al. What caused Earth's temperature variations during the last 800,000 years? Data-based evidence on radiative forcing and constraints on climate sensitivity. Quat. Sci. Rev. 29, 129-145; 9-145 doi: 10.1016/j.quascirev.2009.09.026 (2010).

Martínez-Botí, M. A. et al. Plio-Pleistocene climate sensitivity evaluated using high-resolution CO2 records. Nature 518 , 49-54; doi:10.1038/nature14145 (2015). 
Meraner, K., Mauritsen, T. \& Voigt, A. Robust increase in equilibrium climate sensitivity under global warming. Geophys. Res. Lett. 40, 5944-5948; doi:10.1002/2013GL058118 (2013).

Myhre, G. et al. Anthropogenic and Natural Radiative Forcing Supplementary Material. In: Climate Change 2013: The Physical Science Basis. Contribution of Working Group I to the Fifth Assessment Report of the Intergovernmental Panel on Climate Change [Stocker, T.F. et al. (eds.)]. Available from www.climatechange2013.org and www.ipcc.ch (2013).

Rohling, E.J. et al. Comparing Climate Sensitivity, Past and Present. Annu. Rev. Mar. Sci. 10:12.1-12.28. doi:10.1146/annurev-marine-121916-063242 (2018).

Shao, J. et al. Atmosphere-Ocean CO2 Exchange Across the Last Deglaciation from the Boron Isotope Proxy. Paleoceanography and Paleoclimatology 34, 1650-1670, doi:10.1029/2018pa003498 (2019).

Shao, J. et al. Atmosphere-Ocean CO2 Exchange Across the Last Deglaciation from the Boron Isotope Proxy. Paleoceanography and Paleoclimatology 34, 1650-1670, doi:10.1029/2018pa003498 (2019).

Snyder, C.W. Revised estimates of paleoclimate sensitivity over the past 800,000 years. Climatic Change 156, 121-138; doi:10.1007/s10584-019-02536-0 (2019).

Von der Heydt, A.S. et al. Lessons on Climate Sensitivity from Past Climate Changes. Curr. Clim. Change Rep. 2, 148-158; doi: 10.1007/s40641-016-0049-3 (2016).

Xie, S.-P. Ocean Warming Pattern Effect on Global and Regional Climate Change. AGU Advances 1, e2019AV000130, doi:10.1029/2019av000130 (2020). 